\title{
Ultrasound-guided transbronchial biopsy of solitary pulmonary nodules less than $20 \mathrm{~mm}$
}

\author{
R. Eberhardt*, A. Ernst**\# and F.J.F. Herth*
}

ABSTRACT: Transbronchial biopsy of solitary pulmonary nodules (SPNs) is usually performed under fluoroscopic guidance, but success varies widely. Endobronchial ultrasonography (EBUS) may increase the likelihood of success. The ability of EBUS-guided transbronchial biopsy to sample SPNs of $<20 \mathrm{~mm}$ in diameter was assessed.

All patients seen between June 2004 and August 2007 in whom computed tomography identified a SPN of $<\mathbf{2 0} \mathbf{~ m m}$ underwent bronchoscopic general anaesthesia or moderate sedation for a radial EBUS-guided examination. If a typical ultrasonographic picture of solid tissue could be identified, specimens were taken through a catheter with forceps. If the node was not detected within $20 \mathrm{~min}$, the procedure was terminated.

Of 100 nodules detected in 100 consecutive patients, 67 (mean diameter $15 \mathrm{~mm}$ ) were visualised using EBUS and biopsy specimens taken. A diagnosis was established for 46 (46\%) patients. If the lesion was visualised by EBUS, the diagnostic success was $69 \%$ (46 out of 67 ). The 33 patients whose nodules could not be sampled underwent surgical biopsy. Pneumothorax occurred in three patients.

For SPNs of $<20 \mathrm{~mm}$ that can be detected using ultrasound, EBUS-guided transbronchial biopsy is safe and effective.

KEYWORDS: Bronchoscopy, endobronchial ultrasound, lung cancer, solitary pulmonary nodules, transbronchial lung biopsy

lexible bronchoscopy has a variable and often poor success rate in sampling pulmonary lesions during a normal endobronchial examination. The sensitivity of bronchoscopy for detecting malignancy in a solitary pulmonary nodule (SPN) depends upon the size of the nodule, the proximity of the nodule to the bronchial tree, the success in sampling the nodule and the prevalence of cancer in the study population $[1,2]$. For peripheral lesions of $2.5-4.0 \mathrm{~cm}$ in diameter, a bronchoscopic diagnostic yield of $62 \%$ was reported, but, in the same study, the diagnostic sensitivity in patients with suspicion of cancer decreased to $<40 \%$ if the nodules were $<2.5 \mathrm{~cm}$ in diameter [3]. Successful biopsy is mostly achieved with fluoroscopic guidance, but particularly nodules of $<20 \mathrm{~mm}$ in diameter are difficult or impossible to visualise. Thus, for these nodules, SCHREIBER and MCCRORY [4] found an overall diagnostic sensitivity of $33 \%$ (range 5-76\%) in a metaanalysis, and in only two out of eight studies analysed was the diagnostic yield higher.
Endobronchial ultrasonography (EBUS) with a radial probe is useful in sampling peripheral pulmonary lesions [5-9]. Several articles have reported the success of EBUS-guided transbronchial biopsy in SPNs [5-12], but, in the most of these trials, SPNs of $<20 \mathrm{~mm}$ in diameter were not examined.

An alternative to fluoroscopic guidance is electromagnetic navigation, which can guide the biopsy of peripheral lesions. The reported success in sampling lesions of $<30 \mathrm{~mm}$ in diameter is $\sim 65 \%$ [13-16]. However, electromagnetic navigation is not widely available and requires thinsection computed tomography (CT) for planning and expensive disposables.

CT-guided transthoracic needle aspiration may result in a diagnosis in $74-96 \%$ of patients, again depending on lesion size [17-20], but is associated with reported pneumothorax rates that range $15-44 \%$ [17-21].

The aim of the present study was to investigate whether EBUS-guided transbronchial biopsy could be used successfully in patients with small lesions.

\section{AFFILIATIONS}

*Dept of Pulmonary and Critical Care Medicine, Thoraxklinik-Heidelberg, Heidelberg, Germany.

\#Interventional Pulmonology, Beth Israel Deaconess Medical Center, Boston, MA, USA.

\section{CORRESPONDENCE}

F.J.F. Herth

Dept of Pulmonary and Critical Care Medicine, Amalienstraße 5,

Thoraxklinik

University of Heidelberg

D-69126 Heidelberg

Germany

E-mail: felix.herth@thoraxklinik-

heidelberg.de

Received:

Nov 052008

Accepted after revision:

May 152009

First published online:

May 212009 


\section{PATIENTS AND METHODS}

The present research was conducted in accordance with the 1975 Helsinki Declaration. The study was approved by the institutional review board, and written informed consent was obtained from all patients before bronchoscopy.

Data were collected on consecutive adult patients, who were aged $>40 \mathrm{yrs}$, had a positive smoking history and were referred for diagnostic bronchoscopy. Chest CT scans were reviewed in order to identify all patients with SPNs with a diameter of $<20 \mathrm{~mm}$ and characteristics making a malignant lesion likely (e.g. spiculations and no calcifications present). Standard bronchoscopy was performed, with the patients receiving general anaesthesia or moderate sedation. Several videobronchoscopes (models BF T160 and BF T 180; Olympus, Tokyo, Japan) were used. Radial EBUS guidance was provided by a flexible probe and processor unit (UM-3R, UM-4R and US2020R; Olympus) as described below.

\section{Endobronchial ultrasonography}

A $20-\mathrm{MHz}$ mechanical radial-type probe (UM-S20-20R; Olympus) with an external diameter of $1.7 \mathrm{~mm}$ was inserted through a guide sheath (XB01-836-13; Olympus; external diameter $2.7 \mathrm{~mm}$ ) in the working channel of the videoscope.

The probe was inserted into the bronchi leading to the area in which the lesion was identified on the radiological image. In contrast to the snowstorm-like whitish image of air-containing lung tissue, a solid lesion appears darker and more homogeneous on an ultrasonographic image. Nodules are usually well differentiated against the lung tissue by a bright border because of the difference in impedance (fig. 1). If a typical ultrasonographic image of a nodule could be seen, the probe was removed, but the guide sheath was left in place. Four to six biopsy specimens were obtained through the guide sheath using regular disposable biopsy forceps.

If a nodule was not detected with ultrasound imaging within $20 \mathrm{~min}$, the procedure was stopped. Procedures that detected no nodules or that resulted in a nonspecific bronchoscopic diagnosis of fibrosis or inflammation were considered to be nondiagnostic. All patients with nondiagnostic results were referred for surgical biopsy procedures.

\section{Statistical methods}

The diagnostic sensitivity, specificity and accuracy of the EBUS-guided transbronchial biopsy were calculated using the standard definitions. The $\alpha$ was set at 0.05 , and the SPSS statistical software package was used for all analyses (SPSS version 11.5; SPSS, Chicago, IL, USA).

\section{RESULTS}

From July 2004 to June 2007, 122 consecutive patients with SPNs were screened for the trial. After complete inspection of the bronchial tree, including the subsegmental bronchi, fluoroscopy was performed using a monoplanar C-arm (Suprer 50 CP; Philipps Company, Amsterdam, the Netherlands). If the lesion was visible fluoroscopically, the procedure was continued with transbronchial biopsy in the standard manner, and the patient was excluded from the trial. If the lesion could not be visualised by fluoroscopy, the patient was included in the trial and transbronchial biopsy was attempted under EBUS guidance. Reasons for non-enrolment in 22 cases were fluoroscopically visible lesions (13 patients), a diameter of $>20 \mathrm{~mm}$ (five patients), contraindication for diagnostic bronchoscopy (two patients) or patient refusal of consent (two patients).

Of the 122 screened patients, 100 (44 females and 56 males; mean age 51.7 yrs (range 39-73 yrs)) presented with SPNs of $<20 \mathrm{~mm}$ in diameter. The mean \pm SD diameter of the lesions was $15 \pm 4.2 \mathrm{~mm}$ (range 9-20 mm). Mean nodule size did not differ significantly between detected and nondetected nodules, or between detected nodules that did or did not return a diagnosis (table 1). Most nodules were located in the right and left upper lobes (table 1).

Nodules were localised within $20 \mathrm{~min}$ in 67 (67\%) patients, and a diagnosis was established in 46 out of the 67 (69\%). In the other 21 patients, the lesion was detected and biopsy samples taken, but the pathologist was unable to establish a definitive diagnosis (table 1). Nodules in the right lower and middle lobes were significantly more likely to provide a diagnosis than nodules in other locations (table 1).

Among the $46(46 \%)$ patients in whom transbronchial biopsy was diagnostic, 41 had malignant disease (three metastatic renal cancer, three metastatic breast cancer, three metastatic colon cancer and 32 nonsmall cell lung cancer) and five had benign disease (four sarcoidosis and one tuberculoma).

Of the 21 nodules for which transbronchial biopsy was nondiagnostic, surgical biopsy established that 16 had malignant disease (three metastatic colon cancer, two metastatic renal cancer, one metastatic breast cancer and 10 nonsmall cell

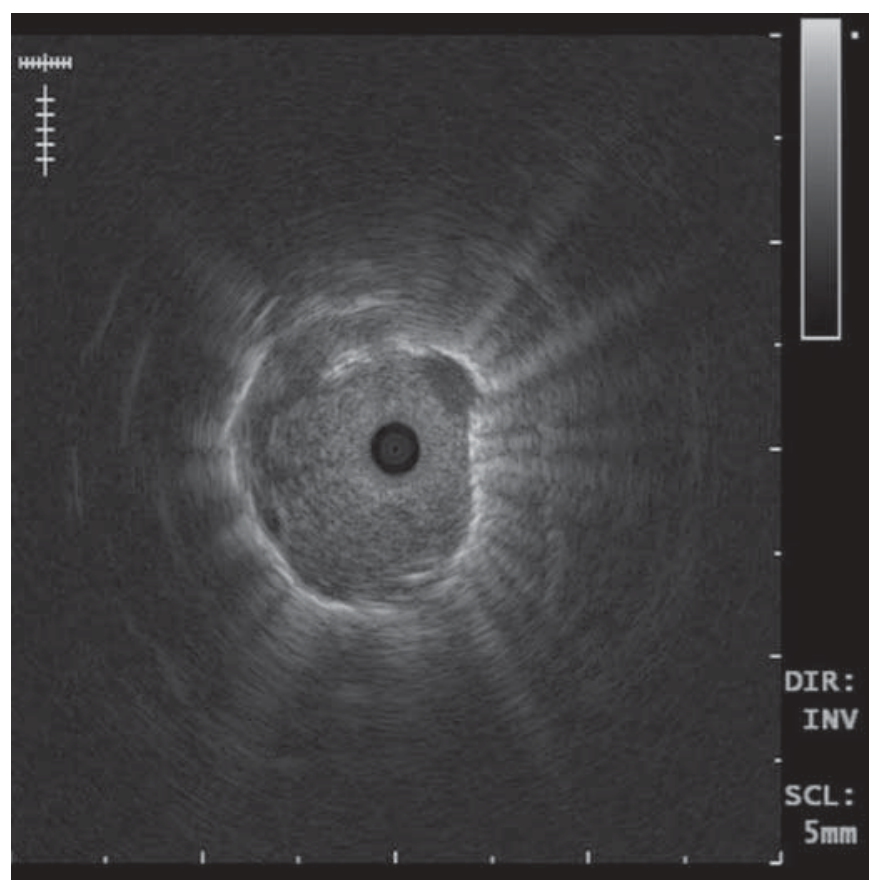

FIGURE 1. Typical endobronchial ultrasonographic image of a single pulmonary nodule, in this case a nodule of $14 \mathrm{~mm}$ in diameter in the left upper lobe of a 53-yr-old male with a suspected diagnosis of lung cancer 


\begin{tabular}{|c|c|c|c|c|c|}
\hline \multirow[t]{3}{*}{ TABLE 1} & \multicolumn{5}{|c|}{$\begin{array}{l}\text { Results of ultrasound-guided transbronchial } \\
\text { biopsy of } 100 \text { single pulmonary nodules of }<20 \\
\text { mm in diameter from } 100 \text { patients }\end{array}$} \\
\hline & \multirow[t]{2}{*}{ Overall } & \multicolumn{2}{|c|}{ Detected } & \multirow[t]{2}{*}{ Not detected } & \multirow[t]{2}{*}{ p-value ${ }^{\#}$} \\
\hline & & Diag & No diag & & \\
\hline Patients n & 100 & 46 & 21 & 33 & \\
\hline $\begin{array}{l}\text { Mean size mm } \\
\text { Lobe } \mathrm{n}(\%)\end{array}$ & 15.3 & 15.7 & 16.0 & 14.5 & 0.28 \\
\hline Right upper & $30(100)$ & $13(43)$ & $7(23)$ & $10(33)$ & 0.13 \\
\hline Right middle & $6(100)$ & $5(83)$ & $1(17)$ & 0 & 0.02 \\
\hline Right lower & $17(100)$ & $10(59)$ & $2(12)$ & $5(29)$ & 0.047 \\
\hline Left upper & $35(100)$ & $12(34)$ & 7 (20) & $16(46)$ & 0.43 \\
\hline Left lower & $12(100)$ & $6(50)$ & $4(33)$ & $2(17)$ & 0.62 \\
\hline
\end{tabular}

lung cancer) and five had benign disease (three chondroid hamartoma, one sarcoidosis and one tuberculoma).

Of 33 patients with SPNs for which transbronchial biopsy could not be performed, surgical biopsy revealed 30 malignant lesions (five metastatic colon cancer, two metastatic renal cancer and 23 nonsmall cell lung cancer) and three benign lesions (two sarcoidosis and one tuberculoma).

The overall diagnostic yield of EBUS-guided transbronchial biopsy was $46 \%$ (46 out of 100). The sensitivity, specificity and negative and positive predicted value for malignancy were 47 , 100, 22 and 100\%. The diagnostic yield for the 67 nodules that were detected with EBUS was $69 \%$ (46 out of 67). The sensitivity, specificity and negative and positive predicted value for malignancy in these cases were $72,100,38$ and $100 \%$ (table 1).

The mean examination time (including the biopsy procedure) was $21.3 \mathrm{~min}$ (range 6-32 $\mathrm{min}$ ). The mean number of specimens obtained from each nodule was 4.2 (range four to six). Self-limited bleeding was observed in three patients; severe bleeding did not occur. Two $(2 \%)$ patients suffered from pneumothorax that was treated with tube thoracostomy. One more was observed, but no intervention was necessary. There were no other complications.

\section{DISCUSSION}

Bronchoscopy has been used to evaluate SPNs and masses for $>30$ yrs. Patients with such nodules frequently undergo transbronchial biopsy under fluoroscopic guidance [22]. However, endoscopic transbronchial biopsy often fails to successfully sample lesions of $<30 \mathrm{~mm}$ or lesions that are fluoroscopically invisible [2, 4, 5]. Nodules too small to be visualised by conventional fluoroscopy usually require additional, often surgical, biopsy procedures.

Therefore, new methods, independent of fluoroscopic visualisation, are needed for navigation and localisation in order to enhance the technical skills of the bronchoscopist. Promising new technologies are electromagnetic navigation and EBUS $[23,24]$.
Several studies [5-12] have reported on the efficacy and safety of EBUS-guided transbronchial biopsy. However, no trial has specifically addressed the yield in patients with nodules of $\leqslant 20 \mathrm{~mm}$ in diameter. This is important information, as with the advent of increased CT imaging and screening, the number of small abnormalities detected is increasing.

In the present study of 100 consecutive patients, our overall diagnostic success rate was $46 \%$ in nodules with a mean diameter of $15 \mathrm{~mm}$. One of the reasons for this low success rate was inability to locate the SPNs with the help of EBUS within $20 \mathrm{~min}$ in 33 patients. For those lesions we were able to detect, the diagnostic success rate was $69 \%$, which is similar to that in other studies [5-12]. This rate may not be as high as it could be since the EBUS location system must be withdrawn from the guide catheter and replaced with the biopsy tool. Replacement can displace the tip of the guide catheter, interfering with the biopsy. This problem becomes more important as the target lesion gets smaller.

Certain aspects of the present study should be acknowledged. It is important to recognise that the prevalence of malignant lesions was high due to the inclusion criteria; therefore, the same results might not be applicable in a more general and unscreened population. One of the limitations of this study is that there was no control group diagnosed by the standard approach of fluoroscopy. We did not use fluoroscopy for navigation since fluoroscopy is known to be unreliable for the identification of small lesions and we wanted to ensure that data would be collected that were specific to one intervention only, namely radial EBUS guidance. Positron emission tomography (PET) results were not taken into consideration as PET imaging was not consistently available at the inception of the study. If performed today, PET results would be taken into consideration, but would not change the findings regarding the performance characteristics of EBUS-guided biopsy.

\section{Conclusions}

For SPNs of $<20 \mathrm{~mm}$ in diameter that can be detected with ultrasound, EBUS-guided transbronchial biopsy is safe and effective. Ultrasound guidance may be more successful than fluoroscopic guidance in sampling small SPNs, increasing the likelihood of a diagnosis and decreasing the need for surgical biopsy. Further studies, potentially combining guided approaches with EBUS, are warranted.

\section{STATEMENT OF INTEREST}

None declared.

\section{REFERENCES}

1 Ost D, Fein AM, Feinsilver SH. Clinical practice: the solitary pulmonary nodule. $N$ Engl J Med 2003; 348: 2535-2542.

2 Baaklini WA, Reinoso MA, Gorin AB, et al. Diagnostic yield of fiberoptic bronchoscopy in evaluating solitary pulmonary nodules. Chest 2000; 117: 1049-1054.

3 Gould MK, Fletcher J, Iannettoni MD, et al. Evaluation of patients with pulmonary nodules: when is it lung cancer? ACCP evidencebased clinical practice guidelines (2nd edition). Chest 2007; 132: Suppl., 108S-130S

4 Schreiber G, McCrory DC. Performance characteristics of different modalities for diagnosis of suspected lung cancer: summary of published evidence. Chest 2003; 123: 115-128. 
5 Herth F, Ernst A, Becker HD. Endobronchial ultrasound guided transbronchial lung biopsy in solitary pulmonary nodules and peripheral lesions. Eur Respir J 2002; 20: 972-974.

6 Shirakawa T, Imamura F, Hamamoto J, et al. Usefulness of endobronchial ultrasonography for transbronchial lung biopsies of peripheral lung lesions. Respiration 2004; 71: 260-268.

7 Paone G, Nicastri E, Lucantoni G, et al. Endobronchial ultrasounddriven biopsy in the diagnosis of peripheral lung lesions. Chest 2005; 128: 3551-3557.

8 Dooms CA, Verbeken EK, Becker HD, et al. Endobronchial ultrasonography in bronchoscopic occult pulmonary lesions. J Thorac Oncol 2007; 2: 121-124.

9 Kurimoto N, Miyazawa T, Okimasa S, et al. Endobronchial ultrasonography using a guide sheath increases the ability to diagnose peripheral pulmonary lesions endoscopically. Chest 2004; 126: 959-965.

10 Herth FJ, Eberhardt R, Becker HD, et al. Endobronchial ultrasound-guided transbronchial lung biopsy in fluoroscopically invisible solitary pulmonary nodules: a prospective trial. Chest 2006; 129: 147-150.

11 Kikuchi E, Yamazaki K, Sukoh N, et al. Endobronchial ultrasonography with guide-sheath for peripheral pulmonary lesions. Eur Respir J 2004; 24: 533-537.

12 Asahina H, Yamazaki K, Onodera Y, et al. Transbronchial biopsy using endobronchial ultrasonography with a guide sheath and virtual bronchoscopic navigation. Chest 2005; 128: 1761-1765.

13 Makris D, Scherpereel A, Leroy S, et al. Electromagnetic navigation diagnostic bronchoscopy for small peripheral lung lesions. Eur Respir J 2007; 29: 1187-1192.

14 Gildea TR, Mazzone PJ, Karnak D, et al. Electromagnetic navigation diagnostic bronchoscopy: a prospective study. Am J Respir Crit Care Med 2006; 174: 982-989.
15 Eberhardt R, Anantham D, Herth FJF, et al. Electromagnetic navigation diagnostic bronchoscopy in peripheral lung lesions. Chest 2007; 131: 1800-1805.

16 Eberhardt R, Anantham D, Ernst A, et al. Multimodality bronchoscopic diagnosis of peripheral lung lesions: a randomized controlled trial. Am J Respir Crit Care Med 2007; 176: 36-41.

17 Kazerooni EA, Lim FT, Mikhail A, et al. Risk of pneumothorax in CT guided transthoracic needle aspiration biopsy of the lung. Radiology 1996; 198: 371-375.

18 Ohno Y, Hatabu H, Takenaka D, et al. CT-guided transthoracic needle aspiration biopsy of small $(\leqslant 20 \mathrm{~mm})$ solitary pulmonary nodules. AJR Am J Roentgenol 2003; 180: 1665-1669.

$19 \mathrm{Li} \mathrm{H}$, Boiselle PM, Shepard J-AO, et al. Diagnostic accuracy and safety of CT-guided percutaneous needle aspiration biopsy of the lung: comparison of small and large pulmonary nodules. AJR Am J Roentgenol 1996; 167: 105-109.

20 Laurent F, Latrabe V, Vergier B, et al. CT-guided transthoracic needle biopsy of pulmonary nodules smaller than $20 \mathrm{~mm}$ : results with an automated 20-gauge coaxial cutting needle. Clin Radiol 2000; 55: 281-287.

21 Laurent F, Michel P, Latrabe V, et al. Pneumothoraces and chest tube placement after CT-guided transthoracic lung biopsy using a coaxial technique: incidence and risk factors. AJR Am J Roentgenol 1999; 172: 1049-1053.

22 Gasparini S, Ferretti M, Secchi EB, et al. Integration of transbronchial and percutaneous approach in the diagnosis of peripheral pulmonary nodules or masses: experience with 1,027 consecutive cases. Chest 1995; 108: 131-137.

23 Shulman L, Ost D. Advances in bronchoscopic diagnosis of lung cancer. Curr Opin Pulm Med 2007; 13: 271-277.

24 Herth FJ, Eberhardt R, Ernst A. The future of bronchoscopy in diagnosing, staging and treatment of lung cancer. Respiration 2006; 73: 399-409. 